\title{
Cerebro-costo-mandibular Syndrome
}

\section{A New Familial Developmental Disorder}

\author{
B. MCNICHOLL, B. EGAN-MITCHELL, J. P. MURRAY, J. F. DOYLE, J. D. KENNEDY, \\ and L. CROME \\ From the Departments of Paediatrics, Radiology, Anatomy and Pathology, Regional Hospital, and \\ University College, Galway, Ireland; and \\ the Department of Neuropathology, Queen Mary's Hospital for Children, Carshalton, Surrey
}

\begin{abstract}
McNicholl, B., Egan-Mitchell, B., Murray, J. P., Doyle, J. F., Kennedy, J. D. and Crome, L. (1970). Archives of Disease in Childhood, 45, 421. Cerebrocosto-mandibular syndrome: a new familial developmental disorder. Three sibs with a hitherto unreported syndrome are described, the main features being mental handicap, palatal defects, micrognathia, and severe costovertebral defects, involving segmentation of most ribs and fusion of their dorsal ends to the vertebral bodies. In addition one infant had hypoplasia of an elbow together with defects of sacrum and coccyx; she and one other sib had minor dental defects. The syndrome is potentially lethal in the neonatal period; one of the sibs has survived. The inheritance is probably autosomal recessive.
\end{abstract}

We describe 3 sibs showing a similar pattern of developmental disorder, involving primarily brain, mandible, ribs, and spine, in which no chromosomal abnormality has been detected. This syndrome does not appear to have been described in sibs. A male infant with several similar features who died at 8 hours, has been described by Smith, Theiler, and Schachenmann (1966); this infant had micrognathia, abnormal tracheal cartilages, and gaps between the ossified dorsal few centimetres of the ribs and the cartilaginous ventral portions.

\section{Case Reports}

Case 1. A firstborn male was delivered by forceps at 41 weeks' gestation in May 1961, weighing $2475 \mathrm{~g}$. The second stage of labour had been prolonged, culminating in some fetal distress. Findings included marked micrognathia, glossoptosis, and a short hard palate ending about $2 \mathrm{~cm}$. from the anterior alveolar margin and having a small central hole. There was no soft palate. The maxilla was thought to be small. Marked respiratory distress was present and the infant was treated in the prone position in an incubator with frequent pharyngeal suction, antibotics, and gavage, but died on the 14th day. Necropsy showed haemorrhagic atelectasis and much aspirated material. It was noted that there were gaps between the posterior portions

Received 3 November 1969. of ribs 4 to 10 inclusive on both sides. The cranial circumference was not recorded and the brain was not examined.

Case 2. A female was born normally at term in January 1963 weighing $2900 \mathrm{~g}$. She showed marked micrognathia, glossoptosis, and a short taut soft palate with absent uvula. There was moderate respiratory distress with inspiratory retraction occurring in the usual lower anterior transverse sulcus, but also in vertical planes posteriorly, parallel to the spine, and producing a hinge-like effect. She was treated similarly to the first infant and suffered repeated respiratory infections during the first year, having considerable glossopharyngo-palatal dysfunction with dysphagia and increased liability to pulmonary aspiration. Laryngoscopy showed an unduly small larynx. She has always had a high-pitched barking cough similar to that which we have heard in children with tracheal stenosis. She had to be tube fed until 15 months, when spoon feeding was gradually introduced. Persistent diarrhoea between 6 and 7 months led to folate deficiency, with mild megaloblastic changes in the bone-marrow and a serum folate of $1.2 \mathrm{ng}$; ; jejunal biopsy was normal. At age 4 years (Fig. 1) she showed prominent eyes and epicanthic folds, small maxillae, micrognathia, and large lowset ears; the middle ears and hearing were grossly normal; the moderate thoracic deformity was apparent. The head circumference at age 6 years was $46 \cdot 6 \mathrm{~cm}$. indicating mild microcephaly; height and weight were $92 \mathrm{~cm}$. and $12 \mathrm{~kg}$., being average measurements for age 


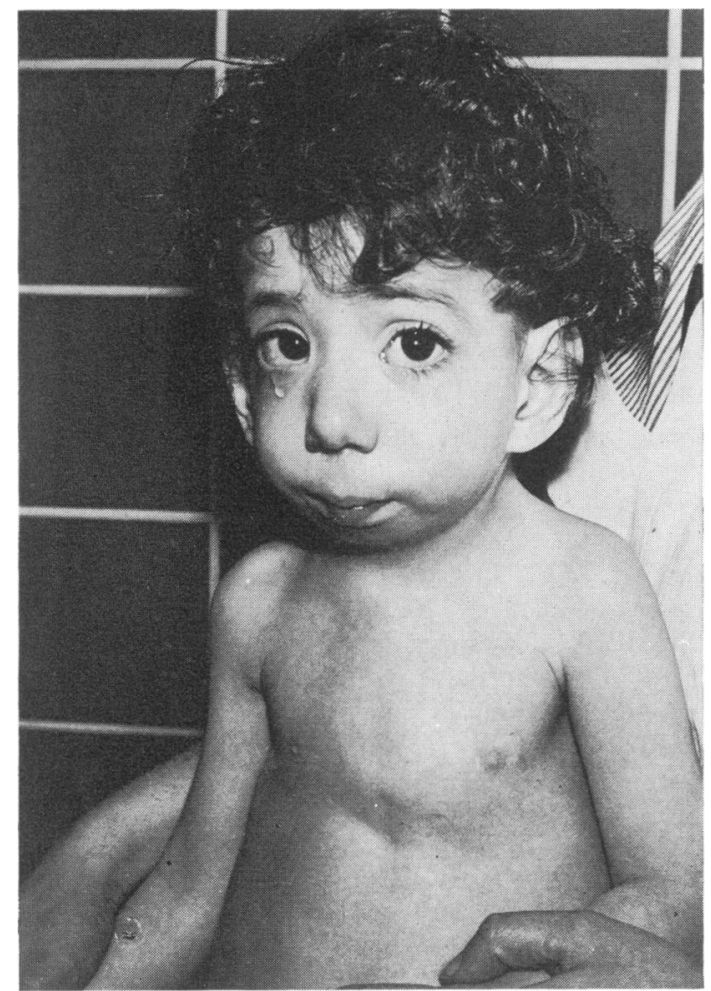

Fig. 1.-Case 2, aged 4 years.

2 years and 10 months, and 2 years respectively; bone age was $3 \frac{1}{2}$ years (Greulich and Pyle, 1959). Then able to walk a little unaided, she had no speech and her mental age was about 1 year. Palmar creases and chromosomal analyses on leucocytes in the second and fourth years were normal, as were several urinary chromatograms.

The major skeletal changes included a normally shaped but small mandible, with absent lower lateral incisors and no corresponding follicles for the permanent ones visible on radiology; partial subluxation of the left elbow was present, with pointed hypoplastic lower end of humerus and upper ends of radius and ulna, the olecranon process of the ulna and the head of the radius being absent; there was mild bilateral coxa valga. Most of the major costal and vertebral abnormalities are shown in Fig. 2; these included minor defects in the neural arches of CV 4 and 5, spina bifida of TV 3, 4, and 5, hypoplastic narrow neural arches of TV 7 and 8 , with absent spinous processes, but a normal neural arch of intervening TV 6 . Lateral views showed anterior wedging of the bodies of TV 2,3,4, and 5, with an acute kyphosis centred on TV 3. Rudimentary ossicles represented the $3 \mathrm{rd}$, 4th, and 5th sacral segments, whereas the 1st and 2nd segments were normal. The most striking feature was the fragmented and rudimen-

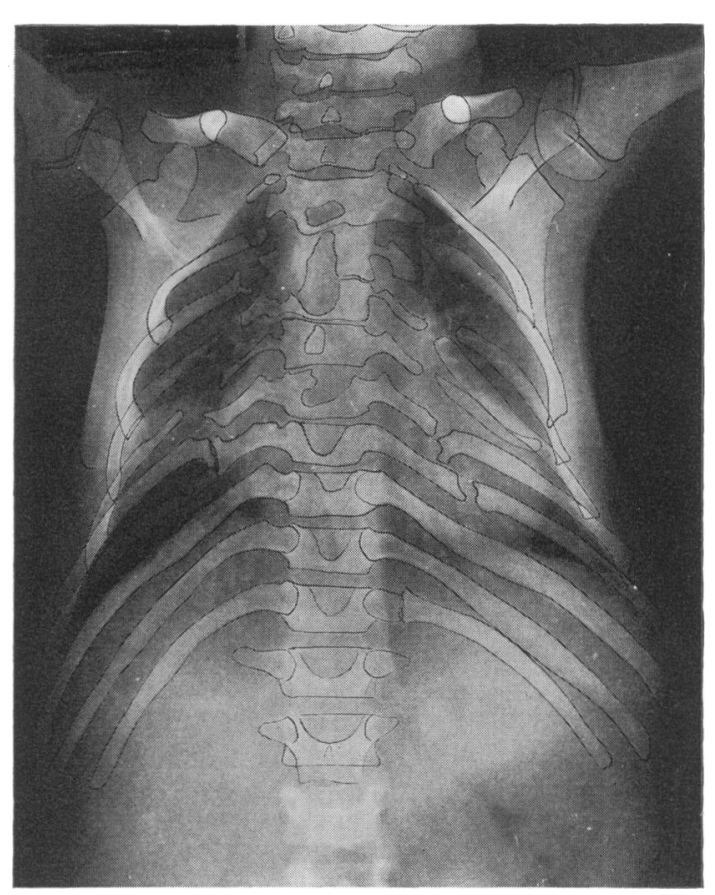

FIG. 2.-X-ray of Case 2 aged 4 years; bony outlines emphasized with ink; some middle lobe collapse-consolidation. Defects described in text. (From Doyle, 1969.)

tary rib development, with fusion of most ribs to the vertebrae and absence of any normal costovertebral articulations. The first left rib was absent (or cartilaginous); ribs 1, 2, and 12 on right, and 3, 6, and 12 on left were rudimentary. Uncalcified gaps were seen between dorsal and ventral segments of ribs 3, 4, 5, 6, and 7 on right and 2,3,4, 5, and 7 on the left. The ventral segment of the 7th right rib was not in alignment with its dorsal segment and was forming a pseudoarthrosis with partial bony bridging to the 8th rib, at a site where a cartilaginous defect had shown on films taken in the neonatal period, the defect being ossified by 4 years. A gap between the left 11 th rib and the costo-transverse process, present in the neonatal period, had been converted to a pseudo-arthrosis by 4 years, and defects in the posterior segments of the left 9th rib and the right 11 th rib had been completely ossified by this time.

Case 3. A male infant was born at 42 weeks' gestation in April 1967, weighing 2920 g. $X$-rays of the infant in utero in February 1967 showed multiple rib deformities and some hydramnios; moderate hydramnios was present at birth. Marked micrognathia and glossoptosis were noted, the hard and soft palates were cleft centrally, and slight epicanthic folds were present. There was severe respiratory distress, the rib defect producing a 'flail' chest with marked 
inspiratory retraction and expiratory grunting. The head circumference was $32.4 \mathrm{~cm} ., 2 \mathrm{SD}$ below the mean (Crome and Stern, 1967); length $49.5 \mathrm{~cm}$. The testicles were undescended. Radiology showed multiple rib defects and poor pulmonary expansion. Management in the prone posture with repeated pharyngeal suction was tried initially, and later a palatal obturator was tried without success. The infant was then intubated, mechanically ventilated, and given intravenous alkali and dextrose, but death occurred at 54 hours. Chromosomal analysis of skin was normal.

Necropsy showed much aspiration and haemorrhagic atelectasis. The brain weighed approximately $307 \mathrm{~g}$., compared with the expected weight of $335 \mathrm{~g}$. for a $2920 \mathrm{~g}$. infant (Crome and Stern, 1967); the approximate value is due to an accident to the cerebellum, the final weight being calculated by adding the expected cerebellar weight. Myelination in general was poor and in particular was absent from the motor corticocapsular tracts and the internal capsule. The nerve cells showed no abnormality, there was no gliosis or other abnormality of cortex, basal ganglia, or white matter. The mandible was small but of normal shape, the follicle for the right first permanent molar was absent; the main costal and vertebral defects are shown in Fig. 3 and 4. Ribs 2 to 9 right and 4 to 9 left were divided into short dorsal and longer ventral segments which were joined by cartilage or fibrous tissues. The total ossified rib

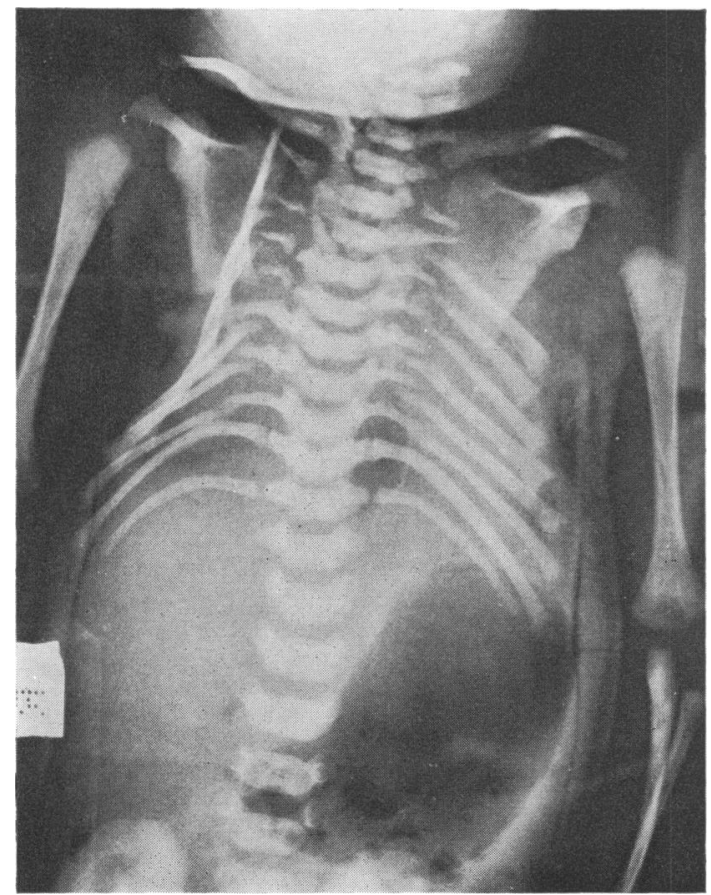

FIG. 3.-Post-mortem X-ray of thorax and abdomen of Case 3 showing characteristic costal defects. (From Doyle, 1969). length was variable and less than usual, the costal cartilages being proportionately long. The dorsal segments extended from the heads to beyond the tubercles, and instead of articulating with the vertebrae showed fusion of the head to the vertebral body. There was also either bony or cartilaginous continuity between rib tubercles and transverse processes, where these had developed. Transverse processes were absent on the right on TV 2, 3, 4, and 5, and on the left on TV 3. Ribs 10 and 11 on both sides were not fragmented and were in continuity with the bodies of TV 10 and 11 . Only rudiments of the 12th rib were found. The sacrum appeared normal on $x$-ray examination but was not included in the dissection of the ribs and spine; the coccyx was not ossified.

\section{Familial Details}

The parents denied any consanguinity. The mother and father were 33 and 40 years of age, respectively, at the birth of the first child (Case 1) in May 1961. Abortions occurred in October 1961 and February 1962, at 6 and 11 weeks. A normal male infant was born in December 1963, at full term, and a further pregnancy resulted in a $6 \frac{1}{2}$-month stillbirth at home in October 1965; this infant was not examined. The parents and the normal sib show no abnormality clinically or on radiology, of the mandible, spine, and thorax, and appear to be of average or better intelligence. No drugs were taken during the early months of the pregnancies.

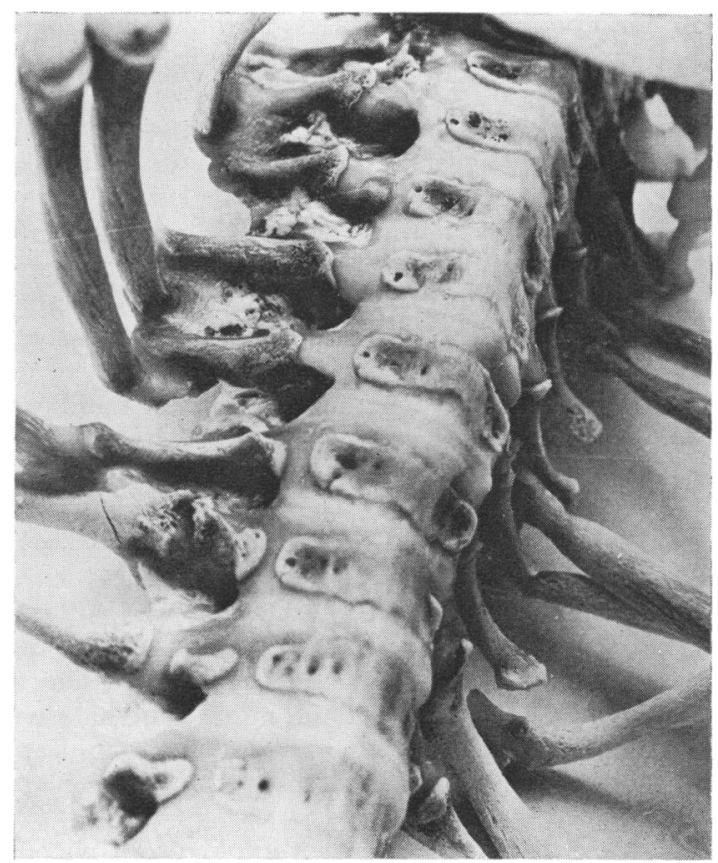

Fig. 4.-Anatomical preparation of part of thorax of Case 3 , showing in particular some rib gaps and fusion of dorsal rib segments to vertebral bodies. (From Doyle, 1969). 


\section{Discussion}

This appears to be a hitherto undescribed familial syndrome due to a genetic defect without detectable chromosomal abnormality. The known major defects in each child correspond, though we have no information about head size in the first. Common to each are abnormalities of the palate, mandible, ribs, and vertebrae. The combination of micrognathia, glossoptosis, palatal dysfunction, and 'flail' chest proved lethal to two infants, though more determined and skilful management might have kept them alive. There are minor variations in the defects of maxilla and mandible, as also in those of the ribs and vertebrae; a defect not present as far as we know in the other infants is the hypoplasia of the left elbow in Case 2.

The infant described by Smith et al. (1966) from Zürich showed many features in common with our cases and may well have had a similar genetic defect. Micrognathia was present in all the cases, but ours did not show webbing of the neck or other redundant areas of skin. The tracheal cartilages were not examined in our 3 infants, but the unusual cough in Case 2 might have been due to abnormalities of the cartilages as described by Smith et al. From a copy of the chest $x$-ray of their case, it appears that the dorsal rib segments fused with the vertebral bodies as in our cases; however, the longer ventral segments of ribs in their case were cartilaginous, whereas they were almost all ossified in ours. It is probable that the basic embryological defect in rib formation was similar in all 4 infants, but their case did not show any mid-line vertebral defects. It is not known whether the Zürich case had any dental or cerebral abnormalities. Lack of reports of this syndrome may be due to rarity, to its lethal nature, or to failure to study the costal and cerebral defects.

Speculation as to whether the mental handicap found in children with mandibulo-glossal defects is due to a congenital cerebral defect or is a sequel of postnatal hypoxia is answered to the extent that our Case 3 had a small brain with delayed myelination of important tracts and would probably have been mentally handicapped had he survived without severe hypoxia. McKenzie (1958) in describing 11 cases of the first-visceral arch syndrome (defects of maxilla, mandible, sphenoid, middle and external ear) mentioned that 2 of them had mental handicap, but did not otherwise discuss the question of cerebral involvement.

Detailed discussion of the embryology and osseous defects will form the subject of a separate paper. Concerning the development of ribs, there are at present two hypotheses; Seno (1961) suggests that the shafts as well as the central part develop from ventral extensions of the somite mesoderm, whereas Straus and Rawles (1953) suggest a separate origin for the shaft in the lateral plate mesoderm. Smith et al. (1966) believe that the separation of dorsal and ventral segments of the ribs in their case is in keeping with separate origins for each segment, but we do not regard their assumption as acceptable. It is quite possible that the segmentation may have been caused by a defect before ossification.

The inheritance of the defect is probably autosomal recessive, in view of the absence of similar congenital defects in the parents. The father has 4 normal sibs who have a total of 17 normal children. The mother has 6 normal sibs, with a total of 30 normal children. One paternal uncle of the father had a 'bad spine' and another was epileptic and probably mentally handicapped, but did not die until his 70s. A maternal first cousin of the father was a deaf-mute. No other congenital defects in the family are known to the parents.

We are grateful to Prof. E. O'Dwyer, under whose care all 3 infants were born, for his encouragement to study them; to Dr. J. Masterton of Dublin and Professor P. E. Polani of London, for the chromosomal analyses of Cases 2 and 3 , respectively; to $\mathrm{Mr}$. A. J. Blayney and Mr. E. G. Hewson for opinions on the larynx and eyes, respectively, of Case 2 ; and to $\mathrm{Mr}$. J. MacNamara for making the palatal obturator for Case 3. We are also indebted to Dr. D. W. Smith of Seattle, Dr. W. C. Grabb of Ann Arbor, and Dr. K. Theiler of Zürich for helpful discussion, and to Dr. A. Giedion of Zürich for a copy of the radiograph of the Zürich infant.

Fig. 2, 3, and 4 originally appeared in an article by Doyle (1969), and are reproduced here by kind permission of the author and publisher.

\section{REFERENCES}

Crome, L., and Stern, J. (1967). Pathology of Mental Retardation, p. 378. Churchill, London.

Doyle, J. F. (1969). The skeletal defects of the cerebro-mandibular syndrome. Irish fournal of Medical Sciences, Series VII, 2, 595.

Greulich, W. W., and Pyle, S. I. (1959). Radiographic Atlas of Skeletal Development of the Hand and Wrist, 2nd ed. Stanford University Press, Stanford, California.

McKenzie, J. (1958). The first arch syndrome. Archives of Disease in Childhood, 33, 477.

Seno, T. (1961). An experimental study on the formation of the body wall in the chick. Acta Anatomica, 45, 60.

Smith, D. W., Theiler, K., and Schachenmann, G. (1966). Rib-gap defect with micrognathia, malformed tracheal cartilages, and redundant skin; a new pattern of defective development. Fournal of Pediatrics, 69, 799.

Straus, W. L., Jr., and Rawles, M. E. (1953). An experimental study of the origin of the trunk musculature and ribs in the chick. American fournal of Anatomy, 92, 471.

Correspondence to Professor B. McNicholl, Regional Hospital, Galway, Ireland. 